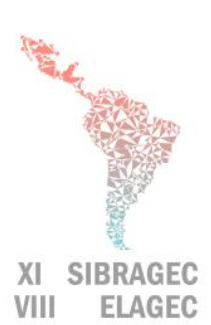

\title{
PRODUTIVIDADE DA MÃO DE OBRA NA EXECUÇÃO DE ALVENARIA ESTRUTURAL EM EDIFÍCIO RESIDENCIAL
}

\section{MULLER (1), Hyago Maurício Bremm; RODEGHERI (2), Priscila Mirapalhete; PALIARI (3), José Carlos}

(1) Programa de Pós-Graduação em Engenharia Civil. Universidade Federal de São Carlos (UFSCar), (66) 99958 6755, hyagomuller@ufscar.br, (2) Programa de Pós-Graduação em Engenharia Civil, UFSCar, priscilamr@ufscar.br, (3) Programa de Pós-Graduação em Engenharia Civil, UFSCar, jpaliari@ufscar.br

\begin{abstract}
The search for improvements in the construction industry has made companies better know their production processes in order to reduce consumption of material and activity that do not add value to the product, as well as ensure better productivity of their teams. For these reasons, this research sought to measure, through a case study, indicators of labor productivity in the execution of the structural masonry of a residential building located in the city of São Carlos, SP, run by a small company. The results showed that the studied team has a higher productivity when compared to the indicators provided by the Sinapi and TCPO tables. In the analysis of time distribution, we observed significantly low unproductive activity and values of good and productive activity and compatible with values presented in the literature. Although it has occurred in a short period of time, this study enlightened that small businesses have optimized their processes in order to remain competitive in the marketplace, demonstrating the efficiency of their team.
\end{abstract}

Keywords: Labor productivity, structural masonry, small companies.

\section{INTRODUÇÃO}

O atual cenário econômico exige que, cada vez mais, as empresas sejam competitivas, entregando os resultados esperados por seus clientes com o mínimo de imprevistos possíveis. Na Indústria da Construção Civil (ICC) não seria diferente, situação agravada após os cortes de investimentos sofridos nos últimos anos. Por esses motivos, as construtoras têm buscado alternativas para reduzir custos e racionalizar a produção, e assim, tornarem-se mais competitivas (SOARES; RODRIGUES; MIRANDA, 2017). Uma das maneiras utilizadas por essas empresas é conhecer melhor seus processos produtivos e com isso reduzir consumo de materiais, etapas que não agregam valor ao produto e revisar as condições de trabalho a fim de garantir melhor produtividade de seus colaboradores.

Este trabalho tem como objetivo avaliar a produtividade da mão de obra na execução de alvenaria estrutural de um edifício residencial situado em São Carlos -SP. Tendo em vista que a empresa é de pequeno porte, assim como mais de $90 \%$ das empresas em atividade da (ICC) no Brasil (SEBRAE-SP, 2018), é relevante se observar a postura desta empresa diante das dificuldade econômicas atuais e como a produtividade da

MULLER, H. M. B.; RODEGHERI, P. M.; PALIARI, J. C. Produtividade da mão de obra na execução de alvenaria estrutural em edifício residencial. In: SIMPÓSIO BRASILEIRO DE GESTÃO E ECONOMIA DA CONSTRUÇÃO, 11., 2019, Londrina. Anais [...]. Porto Alegre: ANTAC, 2019. Disponível em: https://www.antaceventos.net.br/index.php/sibragec/sibragec2019/paper/view/450 
alvenaria estrutural pode ser melhorada, trazendo vantagens competitivas a este estudo de caso.

\section{REVISÃO BIBLIOGRÁFICA}

A produtividade na construção civil foi estudada por diversos autores no Brasil, apresentando conceitos clássicos e aplicando estes a diferentes atividades da construção (OLIVEIRA e SANTOS, 2017; SOARES; RODRIGUES; MIRANDA, 2017; SOUZA, 2000; TRINDADE, 2013). Paliari (2007) apresenta o conceito de produtividade como sendo a relação entre entradas e saídas de um processo, considerando a eficiência da mão de obra em transformar o esforço humano em produtos da construção. Esse autor demonstra os indicadores de Razão Unitária de Produção (RUP) aplicado a equipe diretamente envolvida na atividade em análise. Paravisi (2008) adapta a avaliação de amostragem de trabalho, apresentada por outros autores, a execução de revestimento de fachada predial e debate a importância do entendimento dos tempos produtivos, auxiliares e improdutivos. Filho et al. (2017) realizaram análise da produção científica brasileira de sete anos e constataram que, apesar de entendida a relevância do tema, o número de autores que publicam artigos sobre produtividade em canteiro de obras ainda é relativamente pequeno.

Diante do exposto, entende-se a necessidade de abordar o tema proposto em um estudo de caso típico da ICC brasileira da atualidade. Mesmo o tema não sendo inovador no campo da academia, a relevância de criar uma correlação entre o indicador de uma obra em andamento com os parâmetros apresentados em documentos conceituados na prática da construção civil como TCPO e Sinapi (BRASIL, 2018; CÂMARA BRASILEIRA DO LIVRO, 2010).

\section{MATERIAIS E MÉTODO}

Esta pesquisa possui uma metodologia de objetivo exploratório, desenvolvida por meio de um estudo de caso. Este tipo de procedimento consiste em uma investigação profunda e exaustiva de um ou poucos objetivos, permitindo assim, adquirir conhecimento detalhado sobre o assunto, bem como obter uma visão global do problema ou identificação de possíveis fatores que o influenciam (GIL, 2002).

A unidade-caso escolhida foi uma obra de um edifício residencial localizada no município de São Carlos - SP - BR. O projeto constitui de um prédio com 8 pavimentos tipo e uma garagem, construído em alvenaria estrutural, muito comum na localidade do estudo. Os dados foram coletados entre os meses de julho e agosto de 2018. As medições de produtividade da mão de obra durante a execução da alvenaria estrutural concentraram-se no quarto pavimento tipo, o qual encontrava-se em execução naquele período. Na figura 1 pode ser visto as etapas para o desenvolvimento da pesquisa,

Figura 1 - Etapas do desenvolvimento da pesquisa

\begin{tabular}{|c|c|c|c|}
\hline go & $\begin{array}{l}\text { Seleção da } \\
\text { obra e dos } \\
\text { indicadores }\end{array}$ & $\begin{array}{l}\text { Coleta de } \\
\text { dados } \\
(21 / 07 / 2018 \text { a } \\
17 / 08 / 2018)\end{array}$ & $\begin{array}{l}\text { Análise e } \\
\text { validação } \\
\text { da } \\
\text { amostra }\end{array}$ \\
\hline
\end{tabular}

Fonte: Elaboração própria 
A revisão da bibliografia foi desenvolvida através de pesquisa em bancos de dados científicos por termos como: produtividade na construção, construction productivity, produtividade alvenaria estrutural e productivity in masonry. Os documentos foram filtrados tendo por base sua data de publicação e relevância para os delineamentos da desta pesquisa. Os resultados obtidos apresentaram artigos de periódicos e anais de eventos, dissertações e teses, relatórios técnicos e diversos documentos científicos originários de diferentes partes do mundo.

A pesquisa concentrou-se na obtenção de dois indicadores: de produtividade da mão de obras, expresso pela razão unitária de produção (RUP) proposta por Souza (2000) e o indicador que permite identificar as proporções dos tempos produtivos, improdutivos e auxiliares nos processos, com grau de confiança de $95 \%$ e erro relativo de 5\% (ABROEZEWICZ, 2003). Os dados apresentados nesse trabalho referem-se à produtividade da equipe direta de execução de alvenaria estrutural durante o período de dez dias úteis $\left(\mathrm{RUP}_{\text {direta/diária }}\right)$ enquanto que o indicador relacionado às frações dos tempos foi obtido por observação instantânea, a qual é considerada uma ferramenta estratégica da qualidade total. Ela permite identificar as proporções dos tempos produtivos, improdutivos e auxiliares nos processos, possui grau de confiança de $95 \%$ e erro relativo de 5\% (ABROEZEWICZ, 2003). A metodologia para coleta dos dados dos tempos produtivos baseou-se na pesquisa de Paravisi (2008), com alguns ajustes para a tipologia e a atividade da obra em estudo. Os procedimentos de ambos os indicadores podem ser vistos na figura 2 .

Figura 2 - Procedimentos para obtenção dos indicadores
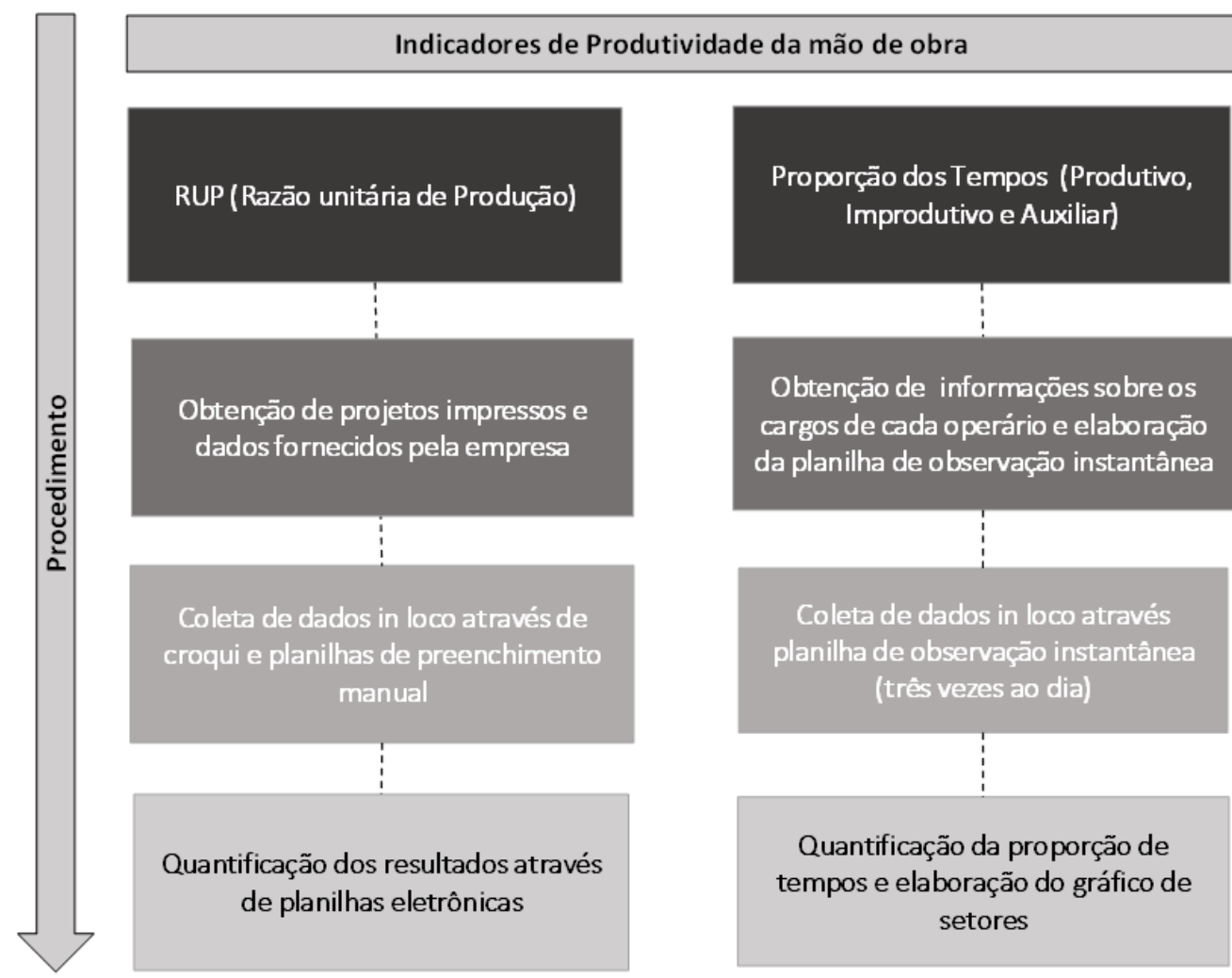

Fonte: Elaboração própria 
SIBRAGEC - ELAGEC 2019 - de 23 a 25 de Outubro - LONDRINA - PR

\section{RESULTADOS}

O Quadro 1 apresenta os dados coletados nos dez dias de acompanhamento das atividades de elevação de alvenaria estrutural na obra estudada através da metodologia proposta por Souza (2000) e Paliari (2007). Neste quadro também são apresentados os valores da RUP cumulativa $_{\text {e RUP }}$ potencial calculados para a equipe direta. É possível verificar que a variação da produtividade diária é significativa, refletindo no distanciamento entre a RUP ${ }_{\text {cumulativa }}$ e RUP potencial $_{\text {para essa atividade. }}$

Quadro 1 - Produtividade da mão de obra de alvenaria estrutural

\begin{tabular}{|c|c|c|c|c|c|c|c|c|c|c|}
\hline \multirow[b]{2}{*}{ Dias } & \multicolumn{2}{|c|}{ Equipe } & \multirow[b]{2}{*}{$\mathrm{Hh}$} & \multirow[b]{2}{*}{$\begin{array}{l}\text { QS } \\
\left(\mathrm{m}^{2}\right)\end{array}$} & \multirow[b]{2}{*}{$\begin{array}{l}\text { RUP } \\
\text { diária }\end{array}$} & \multirow[b]{2}{*}{$\begin{array}{c}\mathrm{Hh} \\
\text { Acum. }\end{array}$} & \multirow[b]{2}{*}{$\begin{array}{c}\text { QS } \\
\text { Acum. } \\
\left(\mathrm{m}^{2}\right)\end{array}$} & \multirow[b]{2}{*}{$\begin{array}{l}\text { RUP } \\
\text { Cum. }\end{array}$} & \multirow{2}{*}{$\begin{array}{c}\text { RUP } \\
\text { diária }< \\
\text { RUP } \\
\text { Cum. } \\
\text { Final }\end{array}$} & \multirow[b]{2}{*}{$\begin{array}{c}\text { RUP } \\
\text { Pot. }\end{array}$} \\
\hline & Qtd & Cargo & & & & & & & & \\
\hline \multirow{2}{*}{1} & 3 & Ofic. & \multirow{2}{*}{24,0} & \multirow{2}{*}{27,04} & \multirow{2}{*}{0,89} & \multirow{2}{*}{24,00} & \multirow{2}{*}{27,04} & \multirow{2}{*}{0,89} & \multirow{2}{*}{$\begin{array}{ll}- & \text { r }\end{array}$} & \\
\hline & 0 & Ajud. & & & & & & & & \\
\hline \multirow{2}{*}{2} & 3 & Ofic. & \multirow{2}{*}{37,6} & \multirow{2}{*}{47,57} & \multirow{2}{*}{0,79} & \multirow{2}{*}{61,60} & \multirow{2}{*}{74,61} & \multirow{2}{*}{0,83} & \multirow{2}{*}{ - } & \\
\hline & 1 & Ajud. & & & & & & & & \\
\hline \multirow{2}{*}{3} & 3 & Ofic. & \multirow{2}{*}{38,0} & \multirow{2}{*}{42,33} & \multirow{2}{*}{0,90} & \multirow{2}{*}{99,60} & \multirow{2}{*}{116,95} & \multirow{2}{*}{0,85} & - & \\
\hline & 1 & Ajud. & & & & & & & - & \\
\hline 4 & 3 & Ofic. & 320 & 9079 & 035 & 13160 & 20774 & 063 & 035 & \\
\hline 4 & 1 & Ajud. & $3,2,0$ & 3 & (נ) & 101,00 & 201,14 & $0,0 \mathrm{~J}$ & $0,5 J$ & \\
\hline 5 & 2 & Ofic. & 270 & 53.02 & 0.51 & 158,60 & 26076 & 0.61 & 0.51 & \\
\hline & 1 & Ajud. & & & & & & & & 0.43 \\
\hline 6 & 2 & Ofic. & 180 & 20.55 & 0.88 & 176,60 & 281.31 & 0.63 & - & \\
\hline & 1 & Ajud. & 10,0 & & & & & & & \\
\hline 7 & 3 & Ofic. & 36.0 & 32.54 & 1.11 & 212.60 & 313.85 & 0.68 & - & \\
\hline & 1 & Ajud. & & & & & & & & \\
\hline 8 & 3 & Ofic. & 36,8 & 36,64 & 1,00 & 249,40 & 350,50 & 0,71 & - & \\
\hline & 1 & Ajud. & 0,0 & 30,04 & 1,00 & $24,+40$ & טנה, & 0,71 & & \\
\hline 0 & 2 & Ofic. & 180 & 2026 & 080 & 26740 & 37075 & 077 & 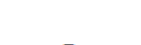 & \\
\hline 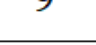 & 1 & Ajud. & 10,0 & 20,20 & 0,03 & 201,40 & 370,73 & 0,72 & - & \\
\hline 10 & 3 & Ofic. & 36.0 & 32,90 & 109 & 303,40 & 403.66 & 0.75 & - & \\
\hline & 1 & Ajud. & (3), & טה, & 1,0 & 300,40 & 400,00 & 0 & 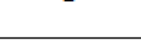 & \\
\hline
\end{tabular}

Fonte: Os autores

A Figura 3 apresenta os resultados obtidos com a aplicação do estudo de tempos conforme proposto por Paravisi (2008). Nele é possível observar que o volume de tempos auxiliares é mais de 50\% do tempo disponível para a execução da atividade, mostrando a necessidade de reavaliação das atividades envolvidas a fim de reduzir essa parcela. 
SIBRAGEC - ELAGEC 2019 - de 23 a 25 de Outubro - LONDRINA - PR

Figura 3 - Distribuição de tempos na execução de alvenaria estrutural

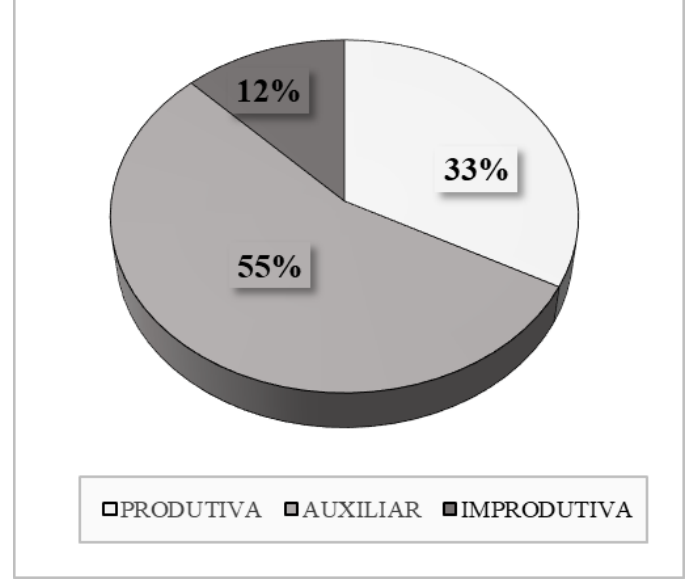

Fonte: Elaboração própria

\section{DISCUSSÃO}

A Figura 4 apresenta os dados de produtividade coletados sobrepostos ao valor de RUP $_{\text {potencial }}$ obtido para este estudo de caso e os valores apresentados na TCPO e Sinapi (BRASIL, 2018; CÂMARA BRASILEIRA DO LIVRO, 2010). A TCPO apresenta a produtividade do item (Alvenaria de blocos para alvenaria estrutural) segregada entre a produtividade do pedreiro e do servente. Para este trabalho, foi utilizada a soma do valor médio da produtividade de um pedreiro e um servente, conforme apresentados na tabela do TCPO. O Sinapi apresenta a composição (Alvenaria de blocos de concreto estrutural 14x19x39 cm, espessura $14 \mathrm{~cm}, \mathrm{Fck}-4,5 \mathrm{MPa}$, para paredes com área líquida maior ou igual a $6 \mathrm{~m}^{2}$ sem vãos, utilizando colher de pedreiro) que mais se aproxima da situação encontrada na obra em estudo, nessa composição também é indicada separadamente a produtividade do pedreiro e do servente, as quais foram somadas para estabelecer o comparativo apresentado.

Figura 4 - Comparativo entre dados coletados em campo e tabelas utilizadas como padrões para construção civil

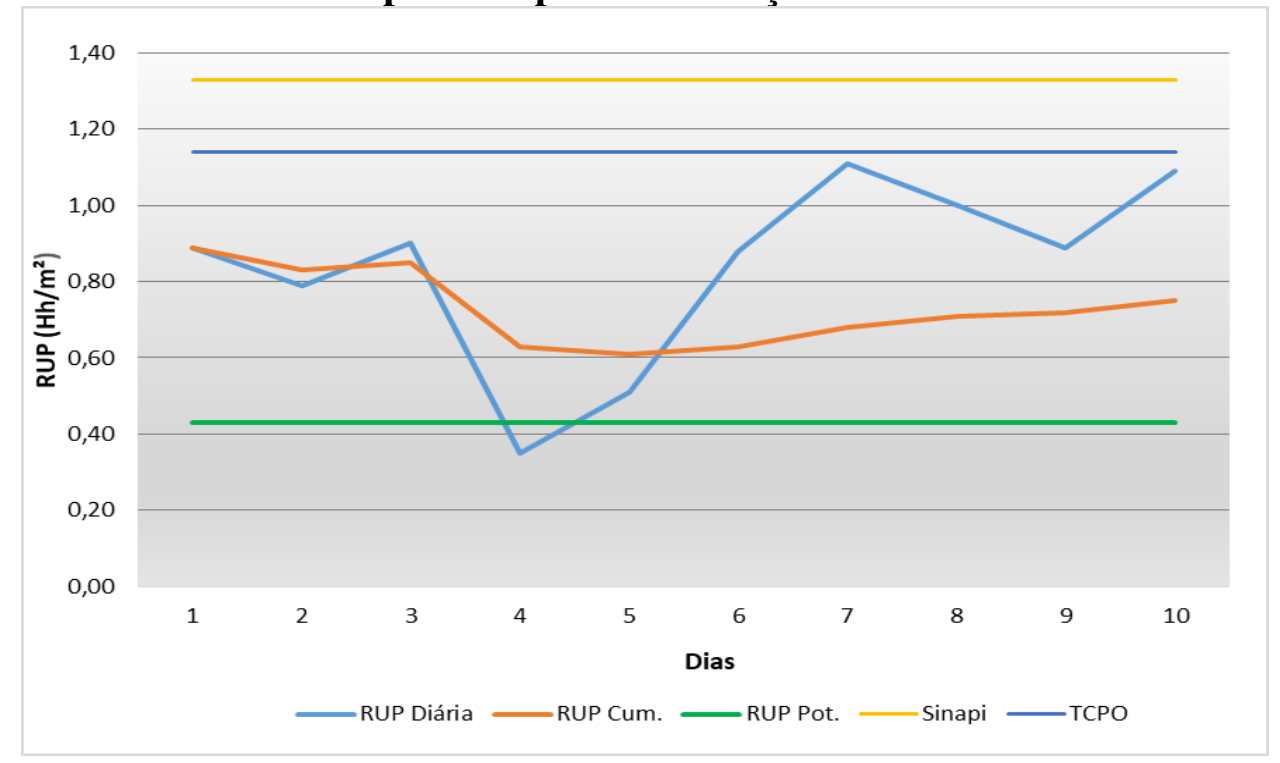

Fonte: Elaboração própria 
SIBRAGEC - ELAGEC 2019 - de 23 a 25 de Outubro - LONDRINA - PR

\section{CONCLUSÕES OU CONSIDERAÇÕES FINAIS}

Através dos dados apresentados, é possível observar que a equipe da obra estudada possui uma produtividade melhor que a média fornecida pelas tabelas TCPO e Sinapi, indicando que a atividade de execução da alvenaria estrutural deste empreendimento possui alto desempenho. Um dos fatores possíveis para este desempenho é o fato de que que provavelmente foram implementadas melhorias desde o início da execução do empreendimento na medida que tenha passado o período de aprendizagem da equipe, fazendo-a mais produtiva.

Para a análise dos tempos, observa-se que 33\% do tempo é efetivamente empregado na produção da alvenaria estrutural, esse valor é compatível com os valores apresentados por Paravisi (2008) e discutidos em seu trabalho, onde afirma que 1/3 do tempo total é aplicado em atividades que agregam valor ao produto final. Isso mostra que mesmo com $55 \%$ do tempo despendido em atividades auxiliares, a equipe consegue atingir a previsão de tempo estimada para a execução efetiva da atividade, sendo a parcela de atividade improdutiva significativamente baixa. Ainda assim, os autores acreditam que um estudo detalhado das condições de trabalho e do canteiro de obras pode influenciar positivamente na distribuição do tempo, tornando parcela do tempo verificados como auxiliares em tempo produtivo.

Este estudo de caso elucida que empresas de pequeno porte têm otimizado seus processos a fim de manterem-se competitivas no mercado, demonstrando a eficiência de suas equipes para atividades significativas a obra (princípio de Pareto). Com os dados apresentados, espera-se uma contribuição ao entendimento da produtividade e distribuição de tempo nas atividades de alvenaria estrutural em empresas brasileiras de pequeno porte.

\section{REFERÊNCIAS}

AMBROZEWICZ, P. H. L. Qualidade na prática: Conceitos e Ferramentas. Curitiba: Serviço Nacional de Aprendizagem Industrial. 2003. Unidade I e Unidade II.

BRASIL, C. E. F. Sistema Nacional de Pesquisa de Custos e Índices da Construção Civil SINAPI. Disponível em: <http://www.caixa.gov.br/poder-publico/apoio-poderpublico/sinapi/Paginas/default.aspx >. Acesso em: 5 maio. 2019.

CÂMARA BRASILEIRA DO LIVRO. Tableas de Composição de Preços para Orçamentos - TCPO. 13 ed. ed. São Paulo - SP - BR: PINI, 2010.

FILHO, W. B. et al. ANÁLISE DA PRODUÇÃo CIENTÍFICA RELACIONADA À

PRODUTIVIDADE EM CONSTRUÇÃO CIVIL. Simpósio Brasileiro de Gestão e Economia da Construção - SIBRAGEC. Anais...Fortaleza - CE - BR: 2017.

GIL, A. C. Como elaborar projetos e pesquisa. 4a ed. São Paulo: Atlas, 2002.

OLIVEIRA, C. A. C.; SANTOS, D. DE G. Redução de variabilidade no processode produção de alvenaria estrutural: padrão técnico e kit completo. Revista Produção Online, v. 17, n. 4, p. 1218-1248, 2017.

PALIARI, J. C. Método para prognóstico da produtividade da mão-de-obra e consumo unitário de materiais: Sistemas prediais hidráulicos. [s.l.] Escola Politécnica da Universidade de São Paulo, 2007.

PARAVISI, S. Avaliação de sistemas de produção de revestimentos de fachada com aplicação mecânica e manual de argamassas. [s.l.] Universidade Federal do Rio Grande do Sul - UFRGS, 2008. 
SIBRAGEC - ELAGEC 2019 - de 23 a 25 de Outubro - LONDRINA - PR

SEBRA-SP. Critérios de classificação de empresas: MEI - ME - EPP, 2018. Disponível em: <http://www.sebrae-sc.com.br/leis/default.asp?vcdtexto=4154> Acesso em: 10 fev. 2019

SOARES, T. B.; RODRIGUES, N. C. S.; MIRANDA, D. A. DE. Análise crítica de indicadores de produtividade e desperdício de material em sistema de alvenaria de vedação racionalizada. Construindo, v. 9, n. 2, p. 1-15, 2017.

SOUZA, U. E. L. DE. Como medir a produtividade da mão-de-obra na construção civil. Encontro Nacional De Tecnologia Do Ambiente Construído - ENTAC. Anais...Salvador - BA BR: 2000. Disponível em: <http://repositorium.sdum.uminho.pt/handle/1822/6799\%5Cngoogle scholar>

TRINDADE, R. DA S. Análise Da Produtividade Da Mão De Obra Na Execução De Alvenaria Estrutural E Alvenaria Convencional. [s.1.] Universidade Federal do Pampa, 2013.

\section{AGRADECIMENTOS}

O presente trabalho foi realizado com apoio da Coordenação de Aperfeiçoamento de Pessoal de Nível Superior- Brasil (CAPES)- Código de Financiamento 001. 